Permanency of relief of tension. In attempting to check a progressive disease or tendency by an outlet of limited capacity, one must feel apprehensive lest the limit of the relief provided be reached sooner or later, unless immediate needs are considerably oversupplied. Unless there were some elasticity in the means provided for relief, more or less immediate hypotony must be a necessary accompaniment of any but quite temporary operative relief of glaucoma. Evidently these filters are not ordinarily called upon to work at once at their full capacity ; some elasticity in action must be admitted (see Trans. Ophth. Soc. U.K., Vol. XXXIX [1919], pp. 227-8).

Also it seems clear that the fundamental changes responsible for the tendency to plus tension-the predisposing causes of glaucoma-commonly progress very slowly, indeed. Otherwise the results of the more moderate filtration operations would be much less durable than they have proved to be. Evidently these operations must act largely by breaking the vicious circle. The most important element in the advance of untreated glaucoma seems to be the high tension itself, as seen in its effect on the filtration angle, and possibly otherwise.

But the practical question of permanence is one to be settled by experience only. One must distinguish between quite early returns of tension (really primary failures to relieve tension satisfactorily) and late recurrence after years. The only examples of the latter known to me, following small flap sclerotomy, or wedge sclerotomy are those mentioned above; but doubtless there must have been others, more particularly in cases which one is inclined now to class as unsuited for sclerotomy.

Here again, as in considering late infection, it is a question of the type of scar produced, rather than of particular operations.

And it may yet prove to be of no small advantage altogether to escape hypotony in providing relief by means of the strictly filtering cicatrix.

The three late failures above mentioned have as yet meant little more than the necessity for supplementary operations. In two of the eyes ample warning was given by the appearance of haloes around lights; and the third eye is still a useful organ.

\title{
THE GOVERNMENT OPHTHALMIC HOSPITAL, MADRAS
}

BY

\section{Norman B. B. Fleming, LONDON.}

DURING the autumn of 1919 , I had the. pleasure and privilege of attending the great eye clinic at the Government Ophthalmic Hospital, Madras. Several others, who have had similar good fortune; have recorded their experience with details of the hospital, its buildings, methods and administration, and a repetition of this from me would, I think, be superfluous; nevertheless, many things have changed and many further improvements been accomplished recently under the able guidance of Colonel Kirkpatrick, who succeeded Coblonel Elliot as superintendent, and a few notes of my impressions may be of interest.

The new infectious block is now in full running order; it is a two-storey building equipped on similar lines to the rest of the hospital and possesses a large theatre provided with two operating tables which are placed each alongside a large .window. The pathological department is much more fully equipped and possesses a wealth of interesting material; Wassermann test and cultures are still made at the King Institute, a very large and up-to-date 
pathological and bacteriological laboratory, presided over by Major Cunningham, who very kindly took me through the Institute and showed me the methods of wholesale vaccine manufacture which he employs there; all other forms of work are done in the hospital laboratory.

The Elliot School of Ophthalmology, in connection with the hospital, was nearing completion when I was there, and I have since heard that it is already in use. It consists of a very large dark room with many cubicles, each equipped with an electric ophthalmoscope and filament lamp, also a theatre fitted for demonstration purposes, a museum, and another dark room for photography.

The routine and efficiency of the subordinate staff, all of whom, with the exception of a few nurses, are Indians, is very remarkable; of this fact the phenomenal amount of work successfully performed bears sufficient testimony.

Two days per week are devoted to intra-ocular operations, one to extra-ocular, and three to the inspection of post-operation cases and dark room work, each day being completed by examining the more important of the external diseases occurring among the out-patients, and a visit to the laboratory.

Certain points in the method of cataract extraction favoured there impressed me. The method of bandaging was to me quite new in practice, though I had seen it described and figured in "Sclero-corneal Trephining." A linen bandage is put on in what might be described as the "ready" position, before the operation is commenced ; it passes round the back of the head, and is provided with a slit on either side for each ear; after the operation the two free ends are folded over the eyes, one passing through a slit in the other and pinned over either temple; two thin strips of the bandage are tied above the head, and two others under the chin. This bandage has the advantage of making the slightest movement of the head quite unnecessary during adjustment, and also it is not easily removed by the wearer-a matter of immense importance in India. The screw of a stop speculum is never used; in fact, it is removed as unnecessary and dangerows. The capsule is opened with a Bowman's needle before the section is made; a T-shaped incision is the rule, the entering needle being half turned to make the horizontal limb, again half-turned to make the vertical limb, and then withdrawn. A corneo-scleral section is always aimed at. The iridectomy is performed with straight scissors. The following manoeuvre is, I think, of great importance and value: for expression, one curette only is used; by means of a little judicious coaxing movement the lens is pushed down from above with one end, and then, with the other, pressure is applied in the ordinary

* R. H. Elliot, 1914, pp. 51-52. 
way on the lower part of the cornea; the lens glides out without the slightest tendency to pass up and catch in the upper angle.

Irrigation is a routine. It is worked by an assistant from a large flask fitted with two tubes and a rubber bulb, on McKeown's system ; a slightly curved-not bent-nozzle is employed, but this is never permitted to enter the wound.

If the patient is at all troublesome a Smith's hook is used during iridectomy, expression, and irrigation. While the operation is proceeding it is distinctly.the exception for any instructions to be given to the patient, but quite the rule for a lively conversation on the patient's family history to be carried on in Tamil or Telegu between him and one of the peons.

I was much interested to see the number of cases of excision of the tarsal plate for old standing trachoma, and the success which followed the procedure. In examining slides of the specimens obtained I was struck by the frequency with which I found isolated portions of the lacrymal gland embedded in the tarsal plates.

In the after treatment of intra-ocular operations the instillation of weak silver nitrate drops is a very favourite and apparently valuable practice; the instillation causes no pain whatever.

The method of illumination practised both for operating and for subsequent examination was recently described by Colonel Kirkpatrick in a letter to the British Journal of Ophthalmology. It would be difficult to over-estimate its efficacy.

Two points of treatment are, I think, worthy of note; one is the use of picric acid drops in vesicular conditions of the cornea and another an eye bath of saturated magnesium sulphate in sloughing corneal ulcers.

I was particularly fortunate in seeing a large number of cases of the epidemic keratitis described by Colonel Kirkpatrick in the British Journal of Obhthalmology of January, 1920, and also the methods by which he endeavoured to elucidate and treat this peculiarly interesting condition.

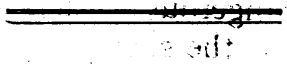

\section{ANNOTATIONS}

\section{Trout and Tar Poisoning}

That trout have totally disappeared from a number of English streams since the war is a fact widely known and puzzling, at all events to fishermen. What is the reason? The popular theory until recently has been that it was due to the contamination of the water with tar products from neighbouring roads and by refuse from 